\title{
À propos de la structuration, de l'enracinement culturel et de la modifiabilité des croyances des enseignants sur le redoublement: synthèse d'un programme de recherche FNS
}

\section{Géry Marcoux, Fanny Boraita et Marcel Crahay}

Dans de nombreux systèmes éducatifs, le redoublement reste en usage avec une forte adhésion des enseignants alors que la littérature scientifique conclut à son inefficacité, voire à ses effets négatifs. Leurs croyances eu égard à cette pratique et leurs résistances aux arguments des chercheurs interpellent. Pour avancer dans la compréhension de ce double constat, différentes recherches ont été menées dans le cadre d'un projet FNS. Elles avaient pour but d'explorer d'une part, la relation des croyances relatives au redoublement avec la connaissance des recherches sur les effets du redoublement ainsi que d'autres catégories de croyances (intelligence, apprentissage, évaluation, justice) et, d'autre part, l'évolution de ces croyances en cours de formation initiale. Pour l'essentiel, les résultats montrent l'influence que peut avoir la connaissance des recherches sur les croyances relatives au redoublement en cours de formation initiale, et, dans une moindre mesure, chez les enseignants en fonction.

\section{Introduction}

Dans de nombreux pays, en particulier, dans les pays francophones, mais aussi dans le Sud de l'Europe (Eurydice, 2011), aux Etats-Unis (NCES, 2009) et en Amérique latine (Institut de Statistique de l'UNESCO, 2012), le redoublement reste en usage avec le fort assentiment des enseignants, et ceci alors que de nombreuses études concluent à l'inefficacité de cette pratique, voire à ses effets négatifs (voir Crahay, 1996, 2004, 2007). Cet état de fait interpelle les chercheurs qui s'étonnent de ce qu'en apparence, les convictions et les pratiques des enseignants résistent aux démonstrations scientifiques en la matière (voir Tanner \& Galis, 1997) ainsi qu'aux tentatives de réforme (voir notamment à ce sujet Crahay \& Donnay, 2001, 2002). Ceci les amène à poser la question: «Mais pourquoi continuent-ils à faire redoubler?» (Marcoux \& Crahay, 2008).

Assurément, le redoublement s'ancre dans une conception traditionnelle de l'école car, comme le soulignent Pouliot et Potvin (2000), «le redoublement est utilisé depuis que l'école existe comme institution sociale, c'est-à-dire depuis que 
les élèves sont regroupés par échelons constituant des barrières qui retiennent les élèves qui n'atteignent pas les objectifs fixés» (p. 49). De surcroit, cette pratique sociale est compatible avec une série de raisonnements et de perceptions relatives aux élèves, à leur façon d'apprendre et à leur «intelligence» qui confortent les enseignants dans l'usage qu'ils font du redoublement face à ceux qui éprouvent des difficultés. Ainsi, constatant que certains élèves n'ont pas réalisé en fin d'année scolaire certains apprentissages jugés fondamentaux pour aborder ceux de l'année qui suit, bon nombre d'enseignants jugent raisonnable de faire recommencer l'année à ceux-ci afin de «consolider les bases» (Crahay, 1996; Haberman \& Dill, 1993). Dans pareil cas, les enseignants utilisent un raisonnement métaphorique: les apprentissages scolaires sont assimilés à la construction d'une maison et il est de notoriété que celle-ci doit reposer sur des fondations solides. Un raisonnement que semble valider l'observation des élèves qui redoublent; il est fréquent, en effet, que les enseignants constatent des progrès chez les redoublants et les attribuent - erronément, du point de vue des chercheurs (voir Smith, 1990) - au fait de répéter l'année scolaire. D'autres fois, les enseignants recourent à des arguments maturationnistes, «invoquant l'âge, la taille ou la corpulence de l'enfant» (Marcoux \& Crahay, 2008, p. 508) ou encore à des arguments renvoyant à des aspects de la nature et du développement des capacités cognitives, comme par exemple, le manque de compréhension (voir Desombre et al., 2010). Bref, comme de nombreuses croyances ${ }^{1}$ relatives à l'éducation, celles concernant le redoublement semblent s'intégrer dans un réseau de croyances psychologiques et pédagogiques (Crahay \& Marcoux, 2010); celles-ci composeraient, pour les enseignants, des sortes de théorie personnelle ou théorie implicite (Vause, 2009) de l'enseignement, de l'apprentissage et du développement. L'imperméabilité de la croyance favorable au redoublement aux conclusions des chercheurs s'expliquerait dès lors par le fait de son intégration dans un réseau d'idées, de convictions et/ou de représentations (Draelants, 2008; Marcoux et Crahay, 2008).

Le programme de recherches mené grâce au soutien du FNS 2 a été conçu pour étudier l'évolution et la structuration (en cours de formation et en cours de carrière) des croyances et connaissances relatives au redoublement, mais aussi les croyances relatives à l'apprentissage, l'intelligence, l'évaluation et les principes de justice. L'hypothèse fondamentale est que les croyances des enseignants sont organisées en réseaux conceptuels, plus ou moins élaborés. Selon cette hypothèse, ce serait le degré d'élaboration de ces réseaux conceptuels qui évolue avec la formation et l'expérience sur le terrain (Crahay \& Marcoux, 2010). 


\section{À propos des croyances des enseignants en fonction}

Les résultats actuellement disponibles concernant la structuration des croyances des enseignants en fonction à propos du redoublement permettent de dégager deux constats majeurs. Premièrement, un lien important apparait entre les croyances à propos du redoublement et la connaissance des recherches sur ses effets. Deuxièmement, les conceptions psychopédagogiques des enseignants affectent peu ces croyances. Ces deux constats sont communs à deux études: une porte sur des enseignants belges de la Province de Liège (Crahay, Marbaise \& Issaieva, 2013); une autre concerne des enseignants suisses romands (Crahay, Issaieva \& Monseur, 2015). Cette convergence mérite d'être soulignée.

Concernant l'étude menée par questionnaire en Province de Liège, il est important de souligner qu'en première analyse, les tendances générales observées convergent avec les résultats des recherches antérieures concernant les croyances des enseignants de la Fédération Wallonie-Bruxelles [FWB] eu égard au redoublement: la majorité des enseignants exprime un avis favorable envers la pratique du redoublement (Crahay, 2007). Cependant, une analyse typologique nuance ce constat. Celui-ci est valable pour une majorité d'enseignants (57 enseignants sur les 112 qui ont répondu, soit $51 \%$ ), mais ne l'est pas pour un certain nombre d'entre eux (39 selon l'analyse typologique, soit 35\%) qui, informés des résultats des recherches sur les effets du redoublement, prennent leur distance par rapport à la croyance commune. Ainsi, il apparait que les enseignants qui peuvent faire état d'une bonne connaissance des recherches scientifiques sur les effets du redoublement déclarent ne plus croire en ses bienfaits. Ceci nous parait être un résultat important et prometteur en termes de changement des mentalités. De ce résultat, on peut déduire l'hypothèse d'action que, si davantage d'enseignants étaient bien informés des recherches sur le redoublement, ils seraient plus nombreux à mettre en doute ses bienfaits. Nous écrivons «bien informés» car les données recueillies révèlent que certains enseignants prétendent connaitre ces recherches, mais leur attribuent des résultats opposés à ce qu'ils sont. Notons encore que, dans notre échantillon, six enseignants attestent d'une réelle connaissance des recherches sur les effets du redoublement et, néanmoins, continuent à croire dans les bienfaits de cette pratique, donc envers et contre les résultats de la recherche. Ceci nous conduit à reconnaitre que l'influence de la connaissance des recherches scientifiques sur les croyances n'est pas automatique (voir Bonvin, Bless \& Schuepbach, 2008; Terry, 2011; Witmer, Hoffman \& Nottis, 2004).

Dans le cadre de l'étude par questionnaires, suisse romande, deux croyances sur le redoublement sont considérées. La première consiste à croire qu'il offre aux élèves en difficulté une seconde chance et, partant, qu'on est en droit d'en attendre des effets positifs quant à leur réussite scolaire. La seconde consiste à penser que le redoublement n'induit pas de préjudices socio-affectifs (voir Range, Holt, Pijanowski \& Young, 2012); le fait de répéter une année n'a pas de 
conséquence négative sur l'estime de soi, ni sur la motivation scolaire. Comme l'ont démontré les résultats de l'analyse factorielle (Crahay, Issaieva \& Monseur, 2015), il est possible de développer certaines croyances relatives à l'efficacité du redoublement indépendamment des croyances quant à ses méfaits potentiels sur le plan affectif. Une analyse par régression multiple indique que seules les conceptions relatives aux fonctions de l'évaluation aident quelque peu à prédire les croyances relatives au redoublement. Le pourcentage net de variance expliquée par ces conceptions est respectivement de $17 \%$ pour la croyance dans les bienfaits scolaires du redoublement et $11 \%$ pour le déni des méfaits socio-affectifs. Par contre, la connaissance des recherches sur les effets du redoublement affecte les deux croyances de façon importante, mais différente. L'effet est plus marqué pour les croyances relatives aux méfaits socio-affectifs qu'il ne l'est pour les croyances relatives à ses bienfaits cognitifs. Cette conclusion repose sur plusieurs résultats. D'une part, Crahay, Issaieva et Monseur (2015) ont pu constater que la corrélation négative entre l'échelle "connaissance» et l'échelle «croyance 2 (déni des méfaits socioaffectifs)» était plus élevée $(\mathrm{r}=-0,62)$ que celle reliant les échelles "connaissance» et «croyance 1 (bienfaits cognitifs)» $(r=-0,29)$. D'autre part, l'analyse de régression vient renforcer ce constat. Les deux échelles "connaissance» et "rapport aux recherches» à elles seules expliquent $24 \%$ de la croyance dans les bienfaits scolaires du redoublement. Ces deux mêmes échelles expliquent $45 \%$ de la variance de la croyance correspondant au déni des méfaits socio-affectifs du redoublement. Il est donc possible de conclure qu'au moins dans le cas des enseignants suisses romands interrogés, la connaissance des recherches sur le redoublement fait davantage reculer le déni des méfaits socio-affectifs de cette pratique éducative que la croyance en ses bienfaits scolaires.

\section{Étude des croyances de futurs enseignants à leur entrée en formation dans différents contextes culturels}

Une étude menée par Boraita et Marcoux (accepté) a permis d'explorer les croyances de futurs enseignants (FE) à leur entrée en formation. Il s'agit, selon ces chercheurs, du meilleur moment pour tester l'hypothèse de l'influence du facteur culturel sur les croyances relatives au redoublement (voir Alkhrisha, 1994) et d'explorer l'idée que la pratique du redoublement s'inscrit dans une culture de l'échec (Crahay, 1996; Perrenoud, 1984). Pour ce faire, Boraita et Marcoux (2011) ont interrogé $1238 \mathrm{FE}$ en tout début de formation initiale dans différentes institutions de formation en Suisse romande $(\mathrm{N}=679)$, France $(\mathrm{N}=150)$ et Belgique francophone $(\mathrm{N}=409)$ en recourant à une échelle mesurant les croyances sur le redoublement, validée préalablement (voir Boraita \& Marcoux, 2013).

Un apport important de cette étude est de conforter les observations réalisées par plusieurs chercheurs auprès d'enseignants en fonction (Bonvin, 2003; 
Marcoux \& Crahay, 2008 ; Smith, 1990) et de futurs enseignants (voir Alkhrisha, 1994; Range, Yonke, \& Young, 2011): la croyance dans les bienfaits du redoublement s'appuie sur une conception maturationniste du développement selon laquelle la répétition d'une année permet de donner du temps à l'élève pour murir.

Un autre apport concerne des variations d'ordre culturel entre les FE français, suisses ou belges qui débutent leur formation. Ainsi, contrairement aux étudiants suisses et français, les FE belges entrent en formation d'enseignant avec l'idée que le redoublement est une pratique normale, voire une bonne pratique, dont la décision repose clairement sur les notes scolaires. L'existence de cultures pédagogiques reflétant les croyances initiales des FE se confirme dans cette étude. Dans le contexte scolaire francophone belge, la culture de la note scolaire et la pratique du redoublement sont fortement ancrées, bien plus que dans les contextes suisses ${ }^{3}$.

Par ailleurs, les résultats de cette étude mettent également en évidence une certaine indépendance dans les croyances concernant l'efficacité du redoublement sur le plan scolaire et celle redoutant ses effets socio-affectifs négatifs. Il apparait que chez certains FE, principalement belges, il semble possible d'adhérer aux croyances dans les bienfaits du redoublement sur le plan scolaire en redoutant ses conséquences socio-affectives et vice-versa. Enfin, il apparait que parmi les FE suisses et français interrogés, on en retrouve presque autant qui ont un profil de croyances plutôt en faveur de la pratique du redoublement qu'en défaveur. Ce n'est que parmi les FE belges que l'on retrouve une tendance à être majoritairement en faveur du redoublement.

\section{Étude de l'effet d'un module de formation sur les croyances de quelques futurs enseignants}

Dans le cadre d'une étude à petite échelle, Boraita (2013) a appliqué un schéma pré-test/traitement/post-test, en proposant deux entretiens semi-structurés à onze étudiants volontaires inscrits en troisième année de formation pour devenir enseignant en Suisse romande avant et après un module de formation incluant des cours relatifs à l'échec scolaire et aux effets du redoublement. Un entretien semi-structuré a été élaboré sur la base des travaux de Marcoux et Crahay (2008) eux-mêmes inspirés d'autres travaux pilotés par Crahay au cours des dernières années (p.ex.: Burdevet, 1994; Fernandez-Fecci \& Granja, 2006; Mastroianni $\&$ Dallafiora, 2007). Les FE ont été interrogés sur leurs croyances relatives au redoublement au regard de leurs autres conceptions psychopédagogiques sur l'apprentissage, l'intelligence, l'évaluation et la justice scolaire. Lors du second entretien, ils ont également été invités à discuter des éléments de la formation qui ont influencé ou non leurs croyances.

Les résultats montrent que, lors du premier entretien, $6 \mathrm{FE}$ sur 11 sont d'emblée défavorables à la pratique, 4 se disent sans avis car ils conçoivent 
le redoublement comme une pratique inhérente au système éducatif et 1 est favorable. Lors du second entretien, les $11 \mathrm{FE}$ disent être contre le redoublement de façon plus nuancée. Cinq d'entre eux évoluent d'une position plutôt contre à une position plus affirmée et les cinq autres changent leurs positions fataliste et favorable vers une opposition à la pratique. Leurs autres conceptions psychopédagogiques se modifient également. De façon générale, les FE s'ouvrent davantage au pari d'éducabilité et renforcent leur conception de l'intelligence évolutive, ils adhèrent plus fortement à l'évaluation formative et à l'égalité des acquis. Il ressort que ce sont uniquement les cours théoriques qui ont influencé les croyances des FE sur le redoublement, en leur permettant notamment d'avoir connaissance des recherches dans ce domaine; les enseignements portant sur les fonctions d'évaluation et plus particulièrement sur la pratique de l'évaluation formative et sur la différenciation sont également désignés comme influents. Leurs conceptions de l'apprentissage, l'intelligence et la justice scolaire sont quant à elles influencées par l'articulation théorie-pratique du module de formation et par la collaboration mise en place entre les $\mathrm{FE}$, les enseignants-formateurs et les enseignants de la formation initiale.

Cette étude met donc en évidence l'influence positive que peut avoir un module de formation initiale sur les croyances de FE; module présentant certaines spécificités sur lesquelles nous reviendrons plus loin. Enfin, il apparait que la croyance dans l'inutilité du redoublement, en plus d'être influencée par la connaissance des recherches sur les effets néfastes de la pratique, est liée à une adhésion à l'égalité des acquis et à l'évaluation formative, des croyances qui engagent clairement le pari d'éducabilité.

Étude de l'évolution des croyances des futurs enseignants au cours de la formation initiale

Dans la foulée de l'étude précédente, Boraita, Crahay et Monseur (en préparation) ont analysé l'effet de la formation initiale sur les croyances de $341 \mathrm{FE}$ primaires relatives au redoublement et tenté de voir comment celles-ci évoluent au regard de la connaissance des recherches et des autres conceptions psychopédagogiques. À défaut de pouvoir mettre en place un design longitudinal, trois cohortes de FE ont été créées afin de tester l'influence de (1) l'entrée en formation et la première année de cours (cohorte 1), (2) la formation focalisée sur l'enseignement primaire (cohorte 2) et (3) la fin de la formation avec la réalisation des stages. Un schéma pré-test/post-test a été appliqué à chacune d'elle, utilisant les questionnaires validés et utilisés dans les précédentes études. La figure 1 donne une représentation du plan de recherche. 


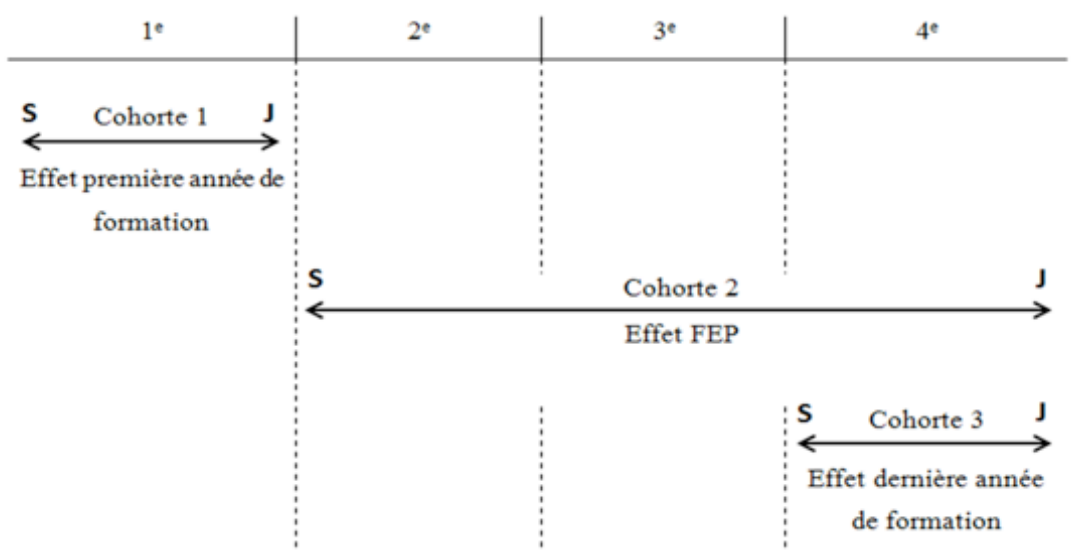

Légende: S: septembre / J: Juin / FEP: Formation Enseignant Primaire

Figure 1: Constitution des différentes cohortes selon l'organisation de la formation d'enseignant primaire en quatre année.

Le premier apport de cette étude est de constater, comme d'autres, une évolution des croyances des FE sur le redoublement (voir Range, Yonke, \& Young, 2011) mais aussi de leurs conceptions psychopédagogiques (c'est-à-dire celles relatives à l'apprentissage, l'intelligence, l'évaluation et la justice scolaire) ainsi que des connaissances sur le redoublement. C'est au cours de la première année de formation que les croyances sur le redoublement évoluent le plus. Cette évolution s'accompagne d'une connaissance des recherches sur les effets de la pratique et semble perdurer en fin de formation. Les conceptions psychopédagogiques évoluent davantage par après, au cours de la formation à l'enseignement primaire et celles-ci s'inscrivent dans le pari d'éducabilité.

Le second apport de cette étude est de confirmer les liens entre la croyance sur le redoublement et, d'une part, la connaissance des recherches, d'autre part, les conceptions psychopédagogiques des FE.

\section{Les croyances sur le redoublement au regard des conceptions psychopédagogiques et de la connaissance des recherches}

Comme le montrent les deux études précédentes, la croyance en défaveur du redoublement paraît liée à des conceptions psychopédagogiques qui engagent le pari d'éducabilité et, assurément, à l'acquisition de connaissances sur les recherches quant aux effets du redoublement. Dans une autre étude menée auprès de $592 \mathrm{FE}$ des quatre années de la formation en Suisse romande, Boraita 
(2015) s'est efforcée de clarifier ces liens. Elle montre que, pour ces étudiants, la croyance dans les bienfaits du redoublement est reliée aux conceptions suivantes: l'intelligence est innée, l'apprentissage se fait par répétition et par déclic, l'évaluation doit être normative et l'école doit respecter une justice scolaire méritocratique. Comme dans les autres recherches présentées, la croyance dans le redoublement est fortement liée à la non-connaissance des recherches sur le redoublement ${ }^{4}$. Inversement, la croyance dans les méfaits du redoublement est reliée, non seulement à la connaissance des recherches scientifiques sur le sujet, mais aussi à des conceptions différentes, souvent inverses: l'intelligence se développe grâce au milieu, l'apprentissage se fait notamment par résolution de problèmes, l'évaluation formative et sommative-critériée constituent des pratiques favorables à l'apprentissage des élèves, l'école doit se fonder sur un principe de justice égalitaire. Des analyses statistiques menées, il ressort que c'est la connaissance des recherches qui explique le plus la croyance relative au redoublement. L'analyse de régression entreprise montre que ce paramètre explique à lui seul plus de $50 \%$ de la variance de la croyance en défaveur du redoublement alors que les conceptions psychopédagogiques ont une influence moindre: elles expliquent moins de 5\% de variance.

Ce dernier résultat va à l'encontre de l'hypothèse formulée au début de nos recherches. Pour rappel, nous posions l'hypothèse fondamentale que les croyances des enseignants sont organisées en réseaux conceptuels, plus ou moins élaborés. Non seulement le résultat de l'analyse de régression calculée par Boraita (2015) ne va pas dans ce sens, mais de surcroît, nous ne pouvons pas, à partir de l'ensemble des recherches menées par notre équipe, dessiner ce que serait le réseau conceptuel sous-jacent à la croyance en défaveur du redoublement. Cependant, à ce stade de nos recherches, il serait prématuré d'exclure l'existence - au moins, chez certains enseignants - de théories pédagogiques en émergence ou ce que l'on pourrait nommer des îlots de rationalité. Des analyses typologiques réalisées dans nos différentes études, il ressort différents profils de croyances parmi les FE genevois interrogés; certains plus cohérents que d'autres. Ceci rejoint d'ailleurs le point de vue de Hofer (2004) selon lequel les étudiants peuvent détenir un ensemble de croyances à travers lesquelles se créent des ponts sans pour autant posséder une théorie globale totalement intégrée et cohérente. L’idée de Hofer est qu'à travers la réflexion et la connaissance, les étudiants ne peuvent alors que coordonner davantage leurs croyances et les faire interagir entre elles.

\section{Le changement de croyances eu égard au redou- blement est-il persistant?}

La question de la persistance des effets de la formation initiale est objet de débats dans la littérature scientifique (voir Haberman \& Dill, 1993). Nombreux sont les auteurs qui soulignent combien la réalité de l'enseignement est complexe (voir 
notamment Huberman, 1987; Perrenoud, 1983). Or, selon Borko et al. (1991, cités dans Kagan, 1992), les formateurs d'enseignants simplifient excessivement la réalité de l'enseignement lorsqu'ils dispensent les cours théoriques. Nombreux sont les FE qui rapportent un manque de liens entre les cours théoriques et leurs expériences de terrain au cours de la formation (Clark \& Peterson, 1986; Duffy \& Roehler, 1986; Olson, 1981; Richardson \& Hamilton, 1994). Selon Aitken et Mildon (1991, cités par Kagan, 1992) ainsi que selon Tillema et Knol (1997), le fossé entre les cours théoriques et les expériences de terrain est tellement grand que les $\mathrm{FE}$ se retrouvent démunis face aux pratiques pédagogiques à mettre en œuvre en classe et se raccrochent alors à leurs propres conceptions ou à celles qu'ils rencontrent sur le terrain. Certains subissent un véritable choc au contact de la réalité de la classe (voir notamment, Huberman, 1987; Veenman, 1984). Il est dès lors légitime de s'interroger sur la stabilité des évolutions de croyances concernant le redoublement que nous avons pu mettre en évidence dans nos études.

L'étude dirigée par Crahay et menée par Hausoul (2005) nourrit cette question car elle montre l'effet fragilisant et déstabilisateur de l'entrée en fonction sur les croyances des FE eu égard au redoublement. Confrontés aux avis d'enseignants chevronnés, des $\mathrm{FE}$ qui ont reçu une formation portant sur les effets du redoublement n'osent pas maintenir leurs croyances autres, nouvellement construites. Plus précisément, Hausoul a pu retrouver 19 des $36 \mathrm{FE}$ qui avaient participé à un module de formation intensive portant sur l'échec scolaire et les effets du redoublement. Elle les a interrogés à nouveau sur leurs croyances en égard au redoublement. Tous ces FE avaient eu l'opportunité d'enseigner pendant les deux années écoulées, ce qui permet à cette étude d'appréhender l'effet de l'insertion professionnelle sur les croyances d'enseignants débutants. Un constat ressort: tous les enseignants qui étaient "pour» ou "plutôt pour» le redoublement à la fin de leur formation ont été renforcés dans leur conviction et pratiquent le redoublement. Pour la plupart de ceux-ci, la réalité du terrain a conforté leur point de vue. Les trois enseignants «mitigés» le sont restés. Pour ceux-ci, le fait de faire redoubler un enfant est bénéfique dans certains cas et pas du tout dans d'autres. Enfin, parmi les enseignants qui étaient "plutôt contre» ou "contre» le redoublement, trois ont changé d'opinion et trois autres sont restés opposés à cette pratique. Cependant, même ces enseignants convaincus de l'inefficacité de la pratique ont, au cours des deux années écoulées, pris des décisions de redoublement. En définitive, cette étude tend à montrer, sur un petit échantillon, que les effets de la formation initiale ne résistent guère aux pressions sociales du milieu au sein duquel doivent s'insérer les jeunes enseignants, soit qu'ils adaptent leurs croyances à celles qui sont dominantes autour d'eux, soit qu'ils maintiennent leurs convictions personnelles tout en agissant comme l'institution attend qu'ils le fassent, ce qui les place dans une situation inconfortable de dissonance cognitive (Festinger, 1957). 
Les résultats de l'étude de Dutrévis, Marcoux, Boraita et Crahay (en préparation) menée auprès d'étudiants en $2^{\mathrm{e}}$ année de formation en enseignement primaire en Suisse romande illustrent l'influence des normes sociales et du poids institutionnel sur les croyances de FE relatives au redoublement. Cette recherche respecte un design expérimental classique en psychologie sociale. Certains sujets ont été interrogés directement sur leurs croyances concernant le redoublement alors que d'autres l'ont été après avoir reçu une information susceptible d'influencer leurs propres réponses: en l'occurrence, il s'agissait de leur donner une information sur le taux d'avis favorables pour un groupe et défavorables pour un autre groupe d'enseignants chevronnés de leur canton. Il apparaît que, lorsqu'on leur demande simplement d'exprimer leur opinion, les FE se montrent plutôt défavorables à la pratique tout en affirmant que les enseignants en fonction seraient, quant à eux, plutôt favorables. En revanche, lorsqu'on induit une norme favorable ou défavorable par rapport aux croyances des enseignants en fonction, les avis des étudiants vont alors dans le même sens que la norme induite. Tout en montrant l'écart entre les croyances des enseignants en fonction et en ceux en formation, ces résultats témoignent de l'influence et du poids des croyances et des avis détenus par les enseignants en fonction sur les enseignants débutants.

\section{Discussion et conclusion}

Alors que la littérature de recherche concernant l'évolution des croyances des FE lors de la formation met en évidence des conclusions plutôt pessimistes (voir Borko \& Putnam, 1996; Richardson \& Placier, 2001), les travaux présentés ici portant spécifiquement sur les croyances relatives au redoublement aboutissent à des constats nettement plus encourageants. Les résultats de l'étude de Boraita (2013) se focalisant sur l'effet d'un module de formation spécifique et l'étude de Boraita, Crahay et Monseur (en préparation) menée auprès de cohortes de FE avant/après des moments clé de leur formation indiquent que les croyances des FE concernant le redoublement peuvent évoluer. En effet, on constate d'une part que les $11 \mathrm{FE}$ interviewés ont tous évolué dans le sens souhaité à la fin du module de formation et rapportent que ce sont les connaissances des recherches qui ont fait évoluer leurs croyances sur le redoublement et d'autre part, que c'est dans la cohorte de FE mesurant l'effet de la première année de cours que la croyance en faveur du redoublement décline le plus alors que la connaissance des recherches augmente le plus.

Ces constats nous paraissent importants: ils nous amènent à nous interroger sur les raisons de cette évolution des croyances. En effet, comment se fait-il que les croyances relatives au redoublement des FE en formation en Suisse romande évoluent en cours de formation alors qu'une partie des recherches dans le domaine conclut de façon opposée? Pour répondre à ces questions, il nous semble nécessaire de revenir sur la revue de la littérature anglo-saxonne réalisée par Boraita et Crahay (2013). 
Il semblerait que le module de formation spécifique suivi par les FE (Boraita, 2013) réponde à certaines des carences de la formation initiale qui feraient obstacle à l'évolution et au changement de croyances au cours de celle-ci. Si le module n'est pas basé sur un travail intensif sur les croyances initiales des FE comme suggéré par les chercheurs du domaine (voir Evans, 1986; Raths, 2001), il s'organise autour d'une articulation de la théorie et de la pratique et d'une collaboration entre les $\mathrm{FE}$, les formateurs de terrain et les formateurs universitaires (voir Kagan, 1992). Les FE peuvent ainsi interagir entre eux et confronter régulièrement leurs points de vue. Ils s'engagent de cette façon dans un processus de réflexion au cours duquel il leur est possible de remettre en cause leurs croyances personnelles et, parallèlement, de s'approprier les apports théoriques des formateurs universitaires. Cette nécessité d'un aller-retour entre la théorie et la pratique est soulignée par les $11 \mathrm{FE}$ de l'étude qualitative et se retrouve dans la littérature de recherche. Nombreux sont les FE qui rapportent un manque de liens entre les cours théoriques et leurs expériences de terrain au cours de la formation (voir Clark \& Peterson, 1986; Olson, 1981; Richardson \& Hamilton, 1994). Les $\mathrm{FE}$ se retrouvent alors démunis face aux pratiques pédagogiques à mettre en ouvre en classe et se raccrochent à leurs propres croyances de l'enseignement (Tillema \& Knol, 1997).

Une autre différence entre les recherches de la littérature et les résultats du programme de recherche présentés ici est l'objet d'étude. L'objet y est ciblé et précis, il s'agit de la pratique du redoublement, alors que les recherches passées en revue par Boraita et Crahay (2013) portent davantage sur des croyances relatives à des phénomènes plus larges tels que les processus d'enseignement, d'apprentissage ou la gestion des élèves. Il s'agit en fait de processus pour lesquels le débat scientifique reste actuel et regorge de positions différentes. Concernant par exemple la question de la gestion de l'hétérogénéité nous avons entendu des FE dire être dans le flou, expliquant «avoir entendu tellement de choses à ce propos» qu'ils ne savent plus se positionner. Concernant le redoublement, toutes les justifications de l'évolution des croyances se rapportent au fait qu'ils ont eu connaissance des recherches. Il s'agit pour les FE de preuves empiriques renforcées par les positions cohérentes de leurs formateurs. De plus, les formateurs des enseignants primaires partagent une position relativement défavorable à la pratique. Les FE se retrouvent donc rarement confrontés à des avis contradictoires dans leur formation universitaire, ce qui n'est probablement pas le cas pour les thématiques relatives à l'enseignement. Comme l'ont souligné différents auteurs (voir Fenstermacher, 1994; Tillema \& Knol, 1997), les incohérences et contradictions entre formateurs d'un même cursus de formation peuvent être un réel obstacle à l'évolution des croyances.

D’une manière générale, les résultats engrangés dans le cadre de ce programme de recherches permettent de dessiner des lignes d'action claires afin de faire évoluer les croyances des enseignants en ce qui concerne le redoublement.

L'influence des connaissances sur les croyances est une donnée essentielle 
pour la formation initiale ou continue étant donné qu'elle témoigne de la nécessité et de l'importance des savoirs théoriques. Que ce soit chez les enseignants en formation ou en fonction (Crahay, Issaieva \& Monseur, 2015; Crahay, Marbaise \& Issaieva, 2013; Pouliot \& Potvin, 2000), les résultats des recherches investiguant ce lien entre connaissances et croyances relatives au redoublement concluent toutes à une influence positive. Suite à Gitlin, Barlow, Burbank, Kauchak et Stevens (1999) qui montrent que les étudiants s'intéressent peu aux recherches, il semble nécessaire que ces savoirs soient transmis pendant des cours obligatoires de la formation initiale. Il est important de donner à tous les FE l'opportunité d'avoir une connaissance approfondie des recherches sur le redoublement. Cette action peut et même doit, selon notre point de vue, s'inscrire dans le cursus de la formation initiale des hautes écoles pédagogiques. Comme l'ont montré pour la Fédération Wallonie-Bruxelles [FWB], les études de Crahay, Ory et Boraita (2012) ainsi que celles de Cantillo Estepa (2009) et de Laduron (2008) dirigées par Crahay, bon nombre de FE terminent leur formation initiale sans avoir entendu parler de ces recherches. Il ressort le même constat des différentes études décrites ci-dessus: parmi les FE suisses romands en fin de formation, certains ont connaissance des recherches sur le redoublement alors que d'autres non. Dès 1995, Bramald, Hardman et Leat pointaient déjà une trop grande diversité entre les cours. De surcroit, il importerait que les formateurs des $\mathrm{FE}$ tiennent tous à propos du redoublement le même langage. À ce propos, dans certaines HEP de Belgique, certains formateurs d'enseignants n'ont pas connaissance de ces recherches. Complémentairement à une action au niveau de la formation initiale, il conviendrait de mener une action d'information à large échelle qui, idéalement, toucherait TOUS les enseignants en fonction car, comme le montre la recherche de Crahay, Marbaise et Issaieva (2013), en Fédération Wallonie-Bruxelles, on trouve encore bon nombre d'enseignants en fonction qui n'ont pas connaissance de ces recherches; un constat que Crahay, Issaieva et Monseur (2015) ont retrouvé avec une moindre ampleur dans le canton de Genève.

Une action conjointe au niveau de la formation initiale des FE et de la formation continuée des enseignants en fonction parait nécessaire. Il convient en effet de rappeler l'étude d'Hausoul (2005) qui a documenté l'effet fragilisant et déstabilisateur de l'entrée en fonction sur les croyances des FE eu égard au redoublement. Confrontés aux avis d'enseignants chevronnés, des FE qui ont reçu une formation portant sur les effets du redoublement, n'osent pas maintenir leurs croyances autres, nouvellement construites. Les résultats de l'étude menée par Dutrévis, Marcoux, Boraita et Crahay (en préparation) vont dans le même sens soulignant l'influence des normes sociales et du poids institutionnel sur les croyances des FE relatives au redoublement.

L'entrée en fonction peut donc bel et bien perturber des convictions engendrées par un dispositif de formation initiale. Les FE genevois ayant participé à l'étude qualitative de Boraita (2013) sont d'ailleurs plusieurs à craindre cette 
épreuve de la réalité. D’autant plus que dans le cas du redoublement, on sait que sur le terrain, il assure une série de fonctions latentes relatives notamment à la gestion de l'hétérogénéité, à la régulation de l'ordre scolaire et au maintien de l'autonomie professionnelle qui servent l'exercice du métier au quotidien (Draelants, 2008).

Ceci indique que la question de la pratique du redoublement n'est pas uniquement du ressort des croyances. Les changer grâce à la connaissance des recherches sur le sujet nous paraît nécessaire, mais certainement pas suffisant. Le changement de croyances chez les enseignants par le biais de la formation initiale ou de formation continue - rappelons qu'à Genève comme en FWB, des enseignants en fonction s'expriment contre le redoublement - ne désamorcera pas automatiquement les fonctions latentes du redoublement (Draelants, 2008) qui amènent les enseignants à le pratiquer tout en déclarant qu'ils le considèrent comme un pis-aller (Marcoux \& Crahay, 2008). Des changements d'ordre institutionnel sont également nécessaires.

\section{Notes}

1 Pour une définition des concepts de "croyance» et "connaissance» nous renvoyons le lecteur aux articles Boraita (2015), Boraita \& Crahay (2013), Crahay, Wanlin, Issaieva \& Laduron (2010), Marcoux \& Crahay (2008).

2 Ce programme de recherche a été mené par l'équipe «Développement Apprentissage et Intervention en Situation Scolaire» [DAISS] de la section des Sciences de l'éducation de l'Université de Genève. Dirigées par le Professeur Marcel Crahay, des études sont menées depuis une vingtaine d'années sur cette thématique et ont débouché sur un Fonds National Suisse réalisé de 2010 à 2014.

3 Concernant les FE français, il convient d'être nuancé car l'échantillon est faible.

4 Notons que, dans cette recherche, Boraita combine les réponses des étudiants des différentes années; ceci explique que certains FE n'aient pas connaissances des recherches sur le redoublement et qu'ils adhèrent à des conceptions sur lesquelles portent des enseignements qui ont pour fonction de les contrarier.

\section{Bibliographie}

Alkhrisha, M. B. (1994). A study of preservice and professional teachers' attitudes toward grade retention. Unpublished doctoral dissertation. University of Cincinnati.

Bonvin, P. (2003). The role of teacher attitudes and judgement in decision-making: the case of grade retention. European Educational Research Journal, 2(2), 277-294.

Bonvin, P., Bless, G. \& Schuepbach, M. (2008). Grade retention: decision-making and effects on learning as well as social and emotional development. School Effectiveness and School Improvement, 19(1), 1-19.

Boraita, F. (2015). Les croyances de futurs enseignants sur le redoublement au regard de leurs connaissances sur ses effets et de leurs conceptions psychopédagogiques. Revue des Sciences de l'Education, 41(3), 483-508.

Boraita, F. (2013). Effet d'un module de formation sur les croyances de futurs enseignants eu égard au redoublement. Etude qualitative à l'Université de Genève. Revue Suisse des Sciences de l'Education, 35(2), 347-369.

Boraita, F. \& Crahay, M. (2013). Les croyances des futurs enseignants: est-il possible de les faire évoluer en cours de formation initiale et, si oui, comment? Revue Française de Pédagogie, 183(2), 99-158. 
Boraita, F. \& Marcoux, G. (2013). Adaptation et validation d'échelles concernant les croyances des futurs enseignants et leurs connaissances des recherches à propos du redoublement. Mesure et Evaluation en Education, 36(1), 49-81.

Boraita, F., Crahay, M. \& Monseur, C. (en préparation). The Effects of Initial Training on the Beliefs Held by Future Teachers with Respect to Grade Retention. Results of a Study Conducted at the University of Geneva.

Boraita, F. \& Marcoux, G. (accepté). Croyances quant à la pratique du redoublement de futurs enseignants entrant en formation dans différents contextes éducatifs. Éducation Comparée, $16(2)$.

Borko, H. \& Putnam, R.-T. (1996). Learning to teach. In D.-L. Berliner \& R.-C. Calfee (Éd.), Handbook of educational psychology (pp. 673-708). New York: MacMillan.

Bramald, R., Hardman, F., \& Leat, D. (1995). Initial teacher trainees and their views of teaching and learning. Teaching and Teacher Education, 11(1), 23-31.

Burdevet, E. (1994). Comment les enseignants prennent-ils une décision de redoublement? Mémoire de licence: Université de Genève.

Cantillo Estepa, C. (2009). Les jeunes enseignants face au redoublement: analyse de l'évolution de leur conception. Mémoire de licence: Université Libre de Bruxelles.

Clark, C. M. \& Peterson, P.-L. (1986). Teachers' thought processes. In M.-C. Wittrock (Éd.), Handbook of research on teaching (3e éd.) (pp. 255-296). New York: Mac Millan.

Crahay, M. (1996). Peut-on lutter contre l'échec scolaire? (1 ${ }^{\mathrm{re}}$ éd.). Bruxelles: De Boeck.

Crahay, M. (2004). Peut-on conclure à propos des effets du redoublement. Revue Française de Pédagogie, 148(1), 11-23.

Crahay, M. (2007). Peut-on lutter contre l'échec scolaire? (3e éd.). Bruxelles: De Boeck.

Crahay, M. \& Donnay, J. (2001). La mise en place de la réforme du premier degré de l'enseignement secondaire. Recherche interuniversitaire, Rapport de recherche auprès du Ministre de l'Enseignement Secondaire, Université de Liège.

Crahay, M. \& Donnay, J. (2002). Où en sont les écoles dans la mise en place du décret relatif à la promotion d'une école de la réussite? Rapport de recherche auprès du Ministre de l'Enseignement Fondamental, Université de Liège.

Crahay, M., Issaieva, E. \& Monseur, Ch. (2015). Les enseignants face au redoublement: ceux qui y croient et ceux qui n'y croient pas. Étude de leurs conceptions psychopédagogiques et de leur connaissance des recherches Revue Française de Pédagogie, 187(2), 35-53.

Crahay, M., Marbaise, C. \& Issaieva, E. (2013). What is teachers' belief in the virtues of student retention founded on? Giornale Italiano della Ricerca Educativa - Italian Journal of Educational Research, 11, 75-94.

Crahay, M. \& Marcoux, G. (2010). Comment et pourquoi les enseignants décident du redoublement de certains élèves? Projet Fond National Suisse de la recherche scientifique [132218] accepté. Berne: Fonds National Suisse de la recherche scientifique.

Crahay, M., Ory, P. \& Boraita, F. (2012). Les croyances des enseignants concernant le redoublement évoluent-elles au cours de leur formation initiale. Annales Scientifiques de la Faculté de Psychologie et Sciences de l'Éducation, de l'Université Ovidius de Constanta, 31-51.

Crahay, M., Wanlin, P., Issaieva, É. \& Laduron, I. (2010). Fonctions, structuration et évolution des croyances (et connaissances) des enseignants. Revue française de pédagogie. Recherches en éducation, 172(3), 85-129.

Desombre, C., Delelis, G., Antoine, L., Lachal, M., Gaillet, F. \& Uraban, E. (2010). Comment des parents d'élèves et des enseignants spécialisés voient la réussite et la difficulté scolaires. Revue Française de Pédagogie, 173(4), 5-18.

Draelants, H. (2008). Les fonctions latentes du redoublement: Enseignements d'une politique de lutte contre le redoublement en Belgique francophone. Education et sociétés, 21(1), 163-180.

Duffy, G. \& Roehler, L. (1986). Constraints on teacher change. Journal of Teacher Education, $35,55-58$. 
Dutrévis, M., Marcoux, G., Boraita, F. \& Crahay, M. (en préparation). Le poids des normes institutionnelles sur les croyances des étudiants en formation eu égard au redoublement.

Eurydice (2011). Le redoublement dans l'enseignement obligatoire en Europe: réglementations et statistiques. Bruxelles: Agence exécutive "Education, Audiovisuel et Culture».

Evans, H. (1986). How do early field experiences influence the student teacher? Journal of Education for Teaching, 12(1), 35-46.

Fenstermacher, G. (1994). The knower and the known: the nature of knowledge in research on teaching. In L. Darling-Hammond (Éd.) Review of research in education, 20, 1-54.

Fernandez-Fecci, A-L. \& Granja, B. (2006). Le redoublement: comment les enseignants en parlent? Etude des conceptions de seize enseignants genevois par rapport au redoublement et comparaison avec les conclusions de Burdevet. Mémoire de licence, Université de Genève.

Festinger, L. (1957). A theory of cognitive dissonance. Stanford (United States): Stanford University Press.

Gitlin, A., Barlow, L., Burbank, M., Kauchak, D. \& Stevens, T. (1999). Pre-service teachers' thinking on research: implications for inquiry oriented teacher education. Teaching and Teacher Education, 15, 753-769.

Haberman, M. \& Dill, V. (1993). The knowledge base on retention vs. teacher ideology: Implications for teacher preparation. Journal of Teacher Education, 44(5), 352-360.

Hausoul E. (2005). Quels sont les effets de l'insertion professionnelle des enseignants sur leurs représentations sociales et, plus précisément, sur leur représentation de l'échec scolaire? Mémoire de licence en sciences de l'éducation, Université de Liège.

Hofer, B.K. (2004). Epistemological Understanding as a Metacognitive Process: Thinking Aloud During Online Searching. Educational Psychologist, 39(1), 43-55.

Huberman, M. (1987). Le cycle de vie professionnelle des enseignants secondaires. Cabiers de la section des sciences de l'éducation de l'université de Genève, 54.

Institut de Statistique de l'UNESCO [ISU] (2012). Opportunités perdues: impact du redoublement et du départ prématuré de l'école. Montréal: ISU. Kagan, D.M. (1992). Professional growth among preservice and beginning teachers. Review of educational research, 62, 129-169.

Laduron, I. (2008). Approche quantitative et exploratoire des croyances psychologiques, épistémologiques et pédagogiques de futurs enseignants du primaire. Mémoire de licence, Université de Liège.

Marcoux, G. \& Crahay, M. (2008). Mais pourquoi continuent-ils à faire redoubler? Essai de compréhension du jugement des enseignants. Revue des Sciences de l'éducation, 30(3), 501-518.

Mastroianni, J. \& Dallafiora, N. (2007). Construction du positionnement des enseignants face au redoublement: propos de 40 enseignants issus des divisions élémentaire et moyenne. Mémoire de Licence, Université de Genève.

National Center for Educational Statistics [NCES]. (2009). The condition of education 2009. Washington, DC: National Center for Educational Statistics. Consulté le 03 juillet 2014 dans http://nces.ed.gov/pubs2009/2009082.pdf

Olson, J. (1981). Teacher influence in the classroom. Instructional Science, 10, 259-275.

Perrenoud, P. (1983). La pratique pédagogique entre l'improvisation réglée et le bricolage. Éducation et recherche, 2, 198-212.

Perrenoud, P. (1984). La fabrication de l'excellence scolaire. Genève: Droz.

Pouliot, L. \& Potvin, P. (2000). Croyances d'enseignants de la maternelle et du primaire à l'égard du redoublement. Revue des Sciences de l'Education, 25(4), 247-261.

Range, B. G., Yonke, D. A. D. \& Young, S. (2011). Preservice teacher beliefs about retention: how do they know what they don't know? Journal of Research in Education, 21(2), 77-99.

Range, B. G., Holt, C.R., Pijanowski, J. \& Young, S. (2012). The perceptions of Primary Grade Teachers and Elementary Principals about the Effectiveness of Grade-Level Retention. The Professional Educator, 36(1), 77-99. 
Raths, J. (2001). Teachers' beliefs and teaching beliefs. Early Childhood Research and Practice, 3(1), 385-391.

Richardson, V. \& Placier, P. (2001). Teacher change. In V. Richardson (Éd.), Handbook of research on teaching (pp. 905-947). Washington DC: American educational research association.

Richardson, V. \& Hamilton, M. L. (1994). The practical argument staff development process. In V. Richardson (Éd.), Teacher change and staff development process: A case in reading instruction (pp. 109-134). New York: Teachers College Press.

Smith, M. L. (1990). Teachers' Beliefs about Retention. In L. A. Shepard \& M. L. Smith (Éd.), Flunking Grades. Research and Policies on Retention (pp. 132-151). Bristol: Falmer Press.

Tanner, K. C. \& Galis, S. A. (1997). Student retention: Why is there a gap between the majority of research findings and school practice? Psychology in the Schools, 34(2), 107-114.

Terry, S. E. (2011). Teachers' beliefs towards grade retention in a rural elementary school. Theses, Dissertations and Capstones. Paper 284. Consulté le 06 septembre 2014 dans http://mds. marshall.edu/etd/284

Tillema, H. \& Knol, W. (1997). Promoting student teacher learning through conceptual change or direct instruction. Teaching and Teacher Education, 13(6), 579-595.

Vause, A. (2009). Les croyances et connaissances des enseignants à propos de l'acte d'enseigner: vers un cadre d'analyse. Les Cahiers de Recherche en Education et Formation. Louvain-laNeuve: Université Catholique de Louvain-la-Neuve.

Veenman, S. (1984). Perceived problems of beginning teachers. Review of Educational Research, 54(2), 143-177.

Witmer, S. M., Hoffman, L. M. \& Nottis, K. E. (2004). Elementary teachers beliefs and knowledge about grade retention: How do we know what they know? Education, 125(2), 173-194.

Mots-clés: Redoublement, croyances des enseignants et futurs enseignants, connaissances de recherches, effet de la formation initiale sur l'évolution des croyances, conceptions psychopédagogiques des enseignants en fonction

\section{Über Strukturierung, kulturelle Verankerung und Veränderbarkeit der Überzeugungen von Lehrpersonen zum Thema Klassenwiederholung: Synthese eines Forschungsprogramms des SNF}

\section{Zusammenfassung}

In vielen Bildungssystemen sind Klassenwiederholungen eine gängige Praxis, die von vielen Lehrpersonen unterstützt wird, obwohl die wissenschaftlichen Erkenntnisse zeigen, dass sie ineffizient sind und sogar negative Effekte erzeugen. Die Überzeugungen der Lehrpersonen zu dieser Praxis und ihre Ablehnung der wissenschaftlichen Argumente werfen Fragen auf. Um diesen Umstand zu verstehen, wurden verschiedene Projekte im Rahmen eines Forschungsprogramms des Schweizerischen Nationalfonds durchgeführt. Dabei wurden zwei Ziele verfolgt. Zum einen wurde der Zusammenhang zwischen den Überzeugungen der Lehrpersonen zur Klassenwiederholung und den wissenschaftlichen 
Erkenntnissen geprüft sowie Überzeugungen zu anderen Kategorien wie Intelligenz, Lernen, Beurteilung und Gerechtigkeit gemessen. Zum anderen wurde die Entwicklung von Überzeugungen während der Grundausbildung untersucht. Die Ergebnisse zeigen, dass die Kenntnisse wissenschaftlicher Ergebnisse zur Klassenwiederholung die Überzeugungen der Lehrpersonen beeinflussen. Dies trifft in höherem Mass für angehende als für amtierende Lehrpersonen zu.

Schlagworte: Klassenwiederholung, Überzeugungen, angehende und amtierende Lehrpersonen, pädagogisch-psychologisches Wissen, Lehrerbildung

\section{A proposito della strutturazione, del radicamento culturale e della modificabilità delle credenze degli insegnanti sulla ripetizione: Sintesi di un programma di ricerca FNS.}

\section{Riassunto}

In molti sistemi educativi, il ricorso alla ripetizione è tuttora in uso, con una forte adesione degli insegnanti, mentre la letteratura scientifica mostra la sua inefficacia, o addirittura i suoi effetti negativi. La fiducia degli insegnanti riguardo a questa pratica e le resistenze a fronte delle argomentazioni dei ricercatori destano importanti interrogativi. Per progredire nella comprensione di questa doppia constatazione, sono state condotte diverse ricerche nell'ambito di un progetto FNS. Esse avevano lo scopo di esplorare da un lato, la relazione fra credenze relative alla ripetizione e conoscenza delle ricerche sui suoi effetti, come pure di altre categorie di credenze (intelligenza, apprendimento, valutazione, giustizia); dall'altro, l'evoluzione di tali credenze nel corso della formazione iniziale. Essenzialmente, i risultati mostrano l'influenza che può esercitare la conoscenza delle ricerche sulle credenze relative alla ripetizione durante la formazione iniziale e, in misura minore, sugli insegnanti in servizio.

Parole chiave: Ripetizione, credenze degli insegnanti e dei futuri insegnanti, conoscenza delle ricerche, effetto della formazione iniziale sulla formazione delle credenze, concezioni psicopedagogiche degli insegnanti in servizio 


\section{Regarding the structuration, cultural rootedness and modifiability of teachers' beliefs concerning grade repetition: Synthesis of a Swiss National Science Foundation research program.}

\section{Summary}

Grade repetition remains in use in many educational systems, with strong backing from teachers, despite an abundant scientific literature drawing attention to its ineffectiveness, if not negative effects. Teachers' beliefs about this practice and their resilience to research claims are puzzling. In an effort to better understand this two-fold observation, several studies have been conducted as part of a FNS research project. Their aim was to explore how beliefs about repetition are related to knowledge about research on the effects of repeating a school year and to other belief categories (intelligence, learning, assessment, justice), on the one hand, and the evolution of these beliefs during pre-service training, on the other. The results of these studies mainly show that knowledge about research findings can have an influence on beliefs about grade repetition during the pre-service teacher training and, to a lesser extent, in the case of in-service teachers.

Keywords: Grade repetition, teachers' and future teachers' beliefs, research knowledge, effect of pre-service training on the evolution of beliefs, in-service teachers' psychological and pedagogical conceptions 